\title{
Kommt jetzt auch noch die elektronische Versichertenkarte?
}

\begin{abstract}
- Bereits am 1.12.2008 haben KBV und GKV-Spitzenverband einen Vertrag zur Einführung der elektronischen Versichertenkarte (eGK) unterschrieben. Demnach erhält jeder Vertragsarzt in einer Einzelpraxis und jede Berufsausübungsgemeinschaft mit bis zu drei Mitgliedern eine Pauschale für den Erwerb eines von der gematik zugelassenen und zertifizierten e-Health-BCS-Kartenterminals.

Vertragsärzte, die nachweisen, dass sie am Notdienst teilnehmen oder Haus-/ Heimbesuche oder Einsätze in Fremdpraxen durchführen, erhalten zusätzlich eine Pauschale für ein mobiles Lesegerät. In einer Ergänzungsvereinbarung wurde nunmehr festgelegt, dass der Zuschuss
\end{abstract}

für das stationäre Gerät 355 Euro, für das mobile Gerät 280 Euro und die notwendigen Installationen in der Praxis 215 Euro beträgt.

\section{MMW Kommentar}

Widerstände bei der Einführung der eGK gibt es zur Zeit nur seitens der Kassenverbände. Diese wollen noch keinen Ausstattungszeitraum festlegen. Das Bundesministerium für Gesundheit (BMG) hat mit einem Änderungsantrag der Fraktionen CDU/CSU und FDP vom 28.10.2010 zum GKV-Finanzierungsgesetz (GKV-FinG) jedoch festgelegt, dass jede Kasse mindestens 10\% ihrer Versicherten bis zum 31.12.2011 mit der eGK ausstatten muss.

\section{Was die aktuelle Bundesregierung unter Kostenerstattung versteht}

- Die christlich-liberale Koalitionsregierung will die Möglichkeit der Kostenerstattung für Versicherte der gesetzlichen Krankenversicherung erleichtern. Deshalb wird ab 2011 die bereits heute bestehende Möglichkeit der Wahl erweitert.

Bisher muss ein GKV-Versicherter seine Krankenkasse vorab in Kenntnis setzen, wenn er das Kostenerstattungs- anstelle des Sachleistungsprinzips praktizieren möchte und ist an diese Wahl ein Jahr gebunden. In Zukunft wird die Bindung für die Kostenerstattung von heute einem Jahr auf drei Monate herabgesetzt. Damit sollen in Zukunft für die Versicherten, die Kostenerstattung wählen, die Folgen deutlich überschaubarer werden, denn weiterhin wird die Kasse für die Versicherten, die Kostenerstattung wählen, nur das erstatten, was sie im Rahmen des Sachleistungsprinzips für diese Leistung erstattet hätte. Der Differenzbetrag ist vom Versicherten zu tragen.

Die Kostenerstattung ist grundsätzlich ein Wahlangebot an die Versicherten. Vor Inanspruchnahme ist die Kasse weiterhin zu informieren.

Damit soll gewährleistet werden, dass der Versicherte nicht kurzfristig durch einen Arzt in eine Kostenerstattung gedrängt werden kann. Die Mindestbindungsfrist für Wahltarife der GKV, mit denen der Krankenversicherungsschutz über das GKV-Niveau abgesichert werden kann, beträgt derzeit drei Jahre und wird auf ein Jahr reduziert. Lediglich bei den sog. Selbstbehalttarifen sowie den Krankengeldtarifen bleibt wegen der bei diesen Tarifen notwendigen langfristigen Kalkulationsgrundlage für die Krankenkassen die bisher geltende Mindestbindungsfrist von drei Jahren erhalten.
Ansonsten droht eine Reduzierung der Verwaltungsausgaben im Jahr 2012 gegenüber dem Jahr 2010 um $2 \%$.

Nach Auffassung der KBV ist deshalb nach einem gewissen Vorlauf zu regionalen Verhandlungen mit den Kassen sowie zur Organisation der Refinanzierung der Geräte ein möglicher Starttermin zum 4. Quartal 2011 denkbar. Kommt es - wie von der KBV ebenfalls geplant - zum 1.7.2010 zur Zwangseinführung der ambulanten Kodierrichtlinien (AKR) und nur ein Quartal später auch noch zur Einführung der eGK, ist schon jetzt absehbar, dass ein geordneter Praxisbetrieb über einen längeren Zeitraum nicht mehr möglich sein wird.

\section{MMW Kommentar}

Die hausärztliche Versorgung dürfte angesichts des dort eher praktizierten Versorgungsprinzips nur wenige GKV-Versicherte veranlassen, dort Kostenerstattung zu wählen.

Dies benachteiligt insbesondere Versichertengruppen, bei denen die Finanzierung der hausärztlichen Versorgung im GKVBereich mangelhaft ist wie z.B. Insassen von Alters- und Pflegeheimen. Dort scheitert eine effektivere und stationäre Behandlungskosten sparende Versorgung meist an der budgetierten Vergütung der Hausbesuche.

Eine Verbesserung der Situation über Kostenerstattungsverträge ist hier nicht zu erwarten, da sich diese Patienten einen solchen Vertrag in der Regel finanziell nicht leisten können. 